\title{
Convex versus Radial Echoendoscopes - Comparison of Capability for Evaluating the Pancreatobiliary Junction
}

\author{
Sung Yong Han and Dong Uk Kim \\ Department of Internal Medicine, Pusan National University School of Medicine and Biomedical Research Institute, Pusan National \\ University Hospital, Busan, Korea
}

See "Capability of Radial- and Convex-Arrayed Echoendoscopes for Visualization of the Pancreatobiliary Junction" by Yoshihide Kanno, Kei Ito, Shinsuke Koshita, et al., on page 274-278.

The echoendoscope (ES), which was developed in the 1980s, is used for the diagnosis and treatment of pancreatobiliary (PB) disease. ${ }^{1-3}$ Endoscopic ultrasonography (EUS) is useful as a surveillance test in asymptomatic patients with high risk for PB malignancies. ${ }^{4,5}$ The failure rate of EUS in diagnosing a PB lesion is known to be about $7 \%,{ }^{6}$ which is lower than the $20 \%$ failure rate for the diagnosis of colonic polyps. However, with a better understanding of the features of this novel equipment, a more accurate method is necessary to reduce the failure rates. Currently, there are two types of ES available, which have slightly different uses depending on their features. The radial ES can visualize structures in the vertical direction with a 360-degree viewing angle, while a convex ES can visualize structures in the parallel direction with a 180-degree viewing angle. Due to this difference, the position of both scopes would be different, when viewing the same structure. It is still debatable as to which scope is better for the diagnosis and treatment of pancreatic malignancies. When convex ES was first introduced, many endosonographers found it difficult to

Received: April 9, 2018 Revised: April 23, 2018

Accepted: April 24, 2018

Correspondence: Dong Uk Kim

Department of Internal Medicine, Pusan National University School of Medicine and Biomedical Research Institute, Pusan National University Hospital, 179 Gudeok-ro, Seo-gu, Busan 49241, Korea

Tel: +82-51-240-7869, Fax: +82-51-254-3237, E-mail: amlm3@hanmail.net ORCID: http://orcid.org/0000-0002-7208-7753

(cc This is an Open Access article distributed under the terms of the Creative Commons Attribution Non-Commercial License (http://creativecommons.org/ licenses/by-nc/3.0) which permits unrestricted non-commercial use, distribution, and reproduction in any medium, provided the original work is properly cited. manage this type of scope because of its narrow field of view. However, with more experience, they are now more comfortable in interpreting the image. Convex ES are now thought to provide a better visualization of the head-body transition region of the pancreas and its vasculature, while radial ES is considered more suitable for the evaluation of ampullary lesions and the gallbladder. ${ }^{6,7}$ However, there are very few published papers, directly comparing radial and convex ES in diagnosing PB malignancies, because of limited available centers and endosonographers skilled in both types of ES.

Kanno and colleagues ${ }^{8}$ studied the comparison of radial and convex ES images at the PB junction, as a retrospective study. In all, 3,644 patients were enrolled in the study, including 1,660 cases with radial ES and 1,984 cases with convex ES. The rate of clear visualization of the PB junction was significantly higher with convex ES than that with radial ES (89.5\% vs. $80 \%$, $p<0.0001)$. Cases in which PB junction was not visualized was $8.4 \%$ and $2.5 \%$ with radial and convex ES, respectively. These results suggested that visualization of the $\mathrm{PB}$ junction using a convex ES was more suitable than with a radial ES. This study showed that the rate of clear visualization would not be similar in evaluating diseases around the ampulla. However, the $\mathrm{PB}$ junction is the most important structure of the ampulla, and if the $\mathrm{PB}$ junction was well visualized, the detection rate of ampullary diseases might increase.

Regarding T staging in PB malignancies, there were several studies which reported similar results between radial and convex ES in the 1990s. ${ }^{9,10}$ Gress and colleagues reported 94\% and $88 \%$ accuracy in $\mathrm{T}$ staging with radial and convex ES, 
respectively. ${ }^{9}$ In a meta-analysis, there was no difference in the accuracy of T staging between radial and convex ES. ${ }^{10}$ In contrast, Jamil and colleagues ${ }^{11}$ reported a significant discrepancy of 38\% in T staging for pancreatic tumors between radial and convex ES, even in a small sample size $(n=13)$. Recently, Kaneko and colleagues ${ }^{7}$ compared radial and convex ES in PB lesions, including the surrounding vessels. Convex ES had better results in visualizing the vascular structure and upper bile duct, and radial ES had better results in visualizing the gallbladder and ampulla lesion. At another site outside the PB lesion, convex ES and radial ES showed similar results; however, convex ES had a longer procedure time (31 min vs. $28 \mathrm{~min}$ ) than radial ES. Shin and colleagues ${ }^{6}$ also compared radial and convex ES in pancreatic lesions. In this study, the failure rate was $9 \%$ for radial ES and $4 \%$ for convex ES during pancreatic cancer screening. Based on recent prospective randomized studies and this study, it was suggested that convex ES is better, compared to radial ES in the evaluation of pancreatic and biliary lesions, except those of the ampulla. However, a largescale randomized controlled study is necessary to confirm this recommendation.

The present study had some limitations. It was a retrospective study and the period of enrolment was different between two groups. Moreover, different ultrasound processors were used between the groups. EU-ME2 (Olympus Co., Tokyo, Japan) was used in the convex group, and EU-ME1 (Olympus Co.) was used in the radial group. Additionally, the proportion of cases that were performed by trainees was slightly higher in the convex group than in the radial group $(735 / 1,984$ [37\%] vs. $537 / 1,660$ [32\%]).

Nevertheless, convex ES might be used as a primary scope for evaluating the $\mathrm{PB}$ junction because it might be superior to radial ES for the diagnosis and surveillance in $\mathrm{PB}$ lesions and could be used for the management of PB diseases.

Conflicts of Interest

The authors have no financial conflicts of interest.

\section{REFERENCES}

1. Maguchi $\mathrm{H}$. The roles of endoscopic ultrasonography in the diagnosis of pancreatic tumors. J Hepatobiliary Pancreat Surg 2004;11:1-3.

2. Mitake M, Nakazawa S, Naitoh Y, et al. Value of endoscopic ultrasonography in the detection of anomalous connections of the pancreatobiliary duct. Endoscopy 1991;23:117-120.

3. Mirbagheri SA, Mohamadnejad M, Nasiri J, Vahid AA, Ghadimi R, Malekzadeh R. Prospective evaluation of endoscopic ultrasonography in the diagnosis of biliary microlithiasis in patients with normal transabdominal ultrasonography. J Gastrointest Surg 2005;9:961-964.

4. Canto MI, Hruban RH, Fishman EK, et al. Frequent detection of pancreatic lesions in asymptomatic high-risk individuals. Gastroenterology 2012;142:796-804; quiz e14-e15.

5. Tanaka M, Fernández-del Castillo C, Adsay V, et al. International consensus guidelines 2012 for the management of IPMN and MCN of the pancreas. Pancreatology 2012;12:183-197.

6. Shin EJ, Topazian M, Goggins MG, et al. Linear-array EUS improves detection of pancreatic lesions in high-risk individuals: a randomized tandem study. Gastrointest Endosc 2015;82:812-818.

7. Kaneko M, Katanuma A, Maguchi H, et al. Prospective, randomized, comparative study of delineation capability of radial scanning and curved linear array endoscopic ultrasound for the pancreaticobiliary region. Endosc Int Open 2014;2:E160-E170.

8. Kanno Y, Ito K, Koshita S, et al. Capability of radial- and convex-arayed echoendoscopes for visualization of the pancreatobiliary junction. Clin Endosc 2018;51:274-278.

9. Gress F, Savides T, Cummings O, et al. Radial scanning and linear array endosonography for staging pancreatic cancer: a prospective randomized comparison. Gastrointest Endosc 1997;45:138-142.

10. Rösch T. Endoscopic ultrasonography in pancreatic cancer. Endoscopy 1994;26:806-807.

11. Jamil LH, Gill KRS, Gross S, et al. Radial versus linear EUS in evaluation of suspected pancreatic cancer: is it sufficient to use linear eus alone? Gastrointest Endosc 2009;69(2 Supple):S266. 